\title{
SURFACE WAVES IN NON-HOMOGENEOUS, GENERAL MAGNETO- THERMO, VISCOELASTIC MEDIA OF HIGHER ORDER
}

\author{
M. SETHI ${ }^{*}$ \\ Govt. Polytechnic, Faculty of Science \\ Jalandharr, INDIA \\ E-mail: munishsethi76@gmail.com \\ K.C. GUPTA \\ Indo-Global College of Engineering \\ Faculty of Science \\ Chandigarh, INDIA \\ M. RANI \\ Faculty of Mathematics \\ gtbkiet, chhapianwali, Malout, INDIA
}

\begin{abstract}
The aim of the present paper is to investigate surface waves in a non-homogeneous, isotropic, visco-elastic solid medium of $n$-th order including the time rate of strain. The theory of generalised surface waves has firstly been developed and then it has been employed to investigate particular cases of waves, viz., Stoneley, Rayleigh and Love type. The wave velocity equations have been obtained for different cases and are in well agreement with the corresponding classical result, when the effects of viscosity, temperature, magnetism as well as nonhomogeneity of the material medium are ignored.
\end{abstract}

Key words: inhomogeneous media, variable density, surface waves, viscosity.

\section{Introduction}

The propagation of surface waves in a non-homogeneous elastic media is of considerable importance in earth-quake engineering and seismology on account of occurrence of non-homogeneities in the earth crust, as the earth is made up of different layers. The theory of surface waves has been developed by Stoneley, Bullen, Ewing et al., Hunters and Jeffreys.

The effect of gravity on wave propagation in an elastic solid medium was first considered by Bromwich (1898), treating the force of gravity as a type of body force. Love (1965) extended the work of Bromwich (1898) and investigated the influence of gravity on superfacial waves and showed that the Rayleigh wave velocity is affected by the gravity field. Sezawa (1927) studied the dispersion of elastic waves propagated on curved surfaces.

The transmission of elastic waves through a stratified solid medium was studied by Thomson. Haskell (1953) studied the dispersion of surface waves in multilayered media. A source on elastic waves is the monograph of Ewing et al. (1957).

Biot (1965) studied the influence of gravity on Rayleigh waves, assuming the force of gravity to create a type of initial stress of hydrostatic nature and the medium to be incompressible. Taking into account, the effect of initial stresses and using Biot's theory of incremental deformations, Dey modified the work of Jones (1964). De and Sengupta (1974) studied many problems of elastic waves and vibrations under the

\footnotetext{
* To whom correspondence should be addressed
} 
influence of gravity field. Sengupta and Acharya (1979) studied the influence of gravity on the propagation of waves in a thermoelastic layer. Brunelle (1973) studied the surface wave propagation under initial tension of compression. Wave propagation in a thin two-layered laminated medium with stress couples under initial stresses was studied by Roy (1984). Datta (1986) studied the effect of gravity on Rayleigh wave propagation in a homogeneous, isotropic elastic solid medium. Goda (1992) studied the effect of non-homogeneity and anisotropy on Stoneley (1924) waves. The interplay of a field with the motion of deformable solids has also been undertaken by many investigators (Knopoff, 1956; Banos, 1956; Chadwick, 1957; Yu and Tang, 1966). $\mathrm{Yu}$ and Tang (1966) discussed the dilatational and rotational waves in a magneto-elastic initially stressed conducting medium. Brunelle studied the surface wave propagation under initial tension of compression. Wave propagation in a thin two-layered laminated medium with stress couples under initial stresses was studied by Roy (1984). Roy and Sengupta investigated the rotatory vibration of a general viscoelastic solid sphere and also the radial vibration of a general viscoelastic solid sphere. The details are found in the work of Eringen and Sahubi (1975). Datta studied the effect of gravity on Rayleigh wave propagation in a homogeneous, isotropic elastic solid medium. Goda studied the effect of non-homogeneity and anisotropy on Stoneley waves. Recently Abd-Alla and Ahmed (1996) studied Rayleigh waves in an orthotropic thermoelastic medium under gravity field and initial stress.

In this work, the problem of $n$-th order viscoelastic surface waves involving the time rate of strain, the medium being isotropic and non-homogeneous has been studied under the influence of a magnetic field and temperature. Biot's theory of incremental deformations has been used to obtain the wave velocity equation for Stoneley, Rayleigh and Love waves. Further these equations are in complete agreement with the corresponding classical results in the absence of viscosity, magnetic and thermal field, non-homogeneity of the material medium.

\section{Formulation of the problem}

Let $M_{1}$ and $M_{2}$ be two non-homogeneous, viscoelastic, isotropic, semi-finite media. They are perfectly welded in-contact to prevent any relative motion or sliding before and after the disturbances and that the continuity of displacement, stress etc. hold good across the common boundary surface. Further the mechanical properties of $M_{1}$ are different from those of $M_{2}$. These media extend to an infinite great distance from the origin and are separated by a plane horizontal boundary and $M_{2}$ is to be taken above $M_{1}$.

Let $O x y z$ be a set of orthogonal Cartesian co-ordinates and let $O$ be any point on the plane boundary and $O z$ points vertically downward to the medium $M_{l}$. We consider the possibility of a type of wave traveling in the direction $O x$, in such a manner that the disturbance is largely confined to the neighborhood of the boundary which implies that the wave is a surface wave.

It is assume that at any instant, all particles in any line parallel to $O y$ having equal displacement and all partial derivatives with respect to $y$ are zero. Further let us assume that $u, v, w$ are the components of displacements at any point $(x, y, z)$ at any time $t$.

The dynamical equations of motion for a three-dimensional non-homogeneous, isotropic, viscoelastic solid medium in Cartesian co-ordinates are

$$
\begin{aligned}
& \frac{\partial \tau_{11}}{\partial x}+\frac{\partial \tau_{12}}{\partial y}+\frac{\partial \tau_{13}}{\partial z}=\rho \frac{\partial^{2} u}{\partial t^{2}} \\
& \frac{\partial \tau_{12}}{\partial x}+\frac{\partial \tau_{22}}{\partial y}+\frac{\partial \tau_{23}}{\partial z}=\rho \frac{\partial^{2} v}{\partial t^{2}} \\
& \frac{\partial \tau_{13}}{\partial x}+\frac{\partial \tau_{23}}{\partial y}+\frac{\partial \tau_{33}}{\partial z}=\rho \frac{\partial^{2} w}{\partial t^{2}}
\end{aligned}
$$


where $\rho$ is the density of the material medium and $\tau_{i j}=\tau_{j i} \forall, i, j$ are the stress components.

Let us consider that the medium is a perfect electric conductor. We take the linearized Maxwell equations governing the electromagnetic field, taking into account the absence of the displacement current (in system-international unit) in the form

$$
\begin{aligned}
& \operatorname{curl} \boldsymbol{h}=\boldsymbol{j}, \\
& \operatorname{curl} \boldsymbol{E}=-\mu_{e} \frac{\partial \boldsymbol{h}}{\partial t}, \\
& \operatorname{div} \boldsymbol{h}=0
\end{aligned}
$$

where

$$
\boldsymbol{h}=\operatorname{curl}\left(\boldsymbol{\mu} \times \boldsymbol{H}_{0}\right),
$$

and

$$
H_{i}=\left(H_{0}\right)_{i}+h_{i}
$$

We consider an orthotropic elastic solid under constant primary magnetic field $\boldsymbol{H}_{0}$ acting on the $y$-axis.

It is assumed that prior to the existence of any disturbance both the media are everywhere at the constant absolute temperature $T_{0}$.

The stress-strain relations for a general isotropic, thermo, viscoelastic medium, according to Voigt are

$$
\tau_{i j}=2 D_{\mu} e_{i j}+\left(D_{\lambda} \Delta-D_{\beta} T+H_{0}^{2} \Delta D_{\mu_{e}}\right) \delta_{i j}
$$

where,

$$
\Delta=\frac{\partial u}{\partial x}+\frac{\partial v}{\partial y}+\frac{\partial w}{\partial z}, D_{\lambda}, D_{\mu}, D_{\beta} \quad \text { are elastic constants. }
$$

Introducing Eq.(2.2) in Eqs (2.1a), (2.1b), (2.1c), we get

$$
\begin{aligned}
& D_{\lambda} \frac{\partial \Delta}{\partial x}+\Delta \frac{\partial D_{\lambda}}{\partial x}+2 D_{\mu} \frac{\partial^{2} u}{\partial x^{2}}+2 \frac{\partial u}{\partial x} \frac{\partial D_{\mu}}{\partial x}-D_{\beta} \frac{\partial T}{\partial x}+D_{\mu} \frac{\partial}{\partial z}\left[\frac{\partial u}{\partial z}+\frac{\partial w}{\partial x}\right]+ \\
& +\left[\frac{\partial u}{\partial z}+\frac{\partial w}{\partial x}\right] \frac{\partial D_{\mu}}{\partial z} H_{0}^{2} D_{\mu_{e}} \frac{\partial \Delta}{\partial x}+H_{0}^{2} \Delta \frac{\partial D_{\mu_{e}}}{\partial x}=\rho \frac{\partial^{2} u}{\partial t^{2}} \\
& D_{\mu} \nabla^{2} v+\frac{\partial v}{\partial x} \frac{\partial D_{\mu}}{\partial x}+\frac{\partial v}{\partial z} \frac{\partial D_{\mu}}{\partial z}=\rho \frac{\partial^{2} v}{\partial t^{2}}, \\
& D_{\mu} \frac{\partial}{\partial x}\left(\frac{\partial u}{\partial z}+\frac{\partial w}{\partial x}\right)+\left(\frac{\partial u}{\partial z}+\frac{\partial w}{\partial x}\right) \frac{\partial D_{\mu}}{\partial x}+2 D_{\mu} \frac{\partial^{2} w}{\partial z^{2}}+2 \frac{\partial w}{\partial z} \frac{\partial D_{\mu}}{\partial z}+ \\
& +D_{\lambda} \frac{\partial \Delta}{\partial z}+\Delta \frac{\partial D_{\lambda}}{\partial z}-D_{\beta} \frac{\partial T}{\partial z}-T \frac{\partial D_{\beta}}{\partial z} H_{0}^{2} D_{\mu_{e}} \frac{\partial \Delta}{\partial z}+H_{0}^{2} \Delta \frac{\partial D_{\mu_{e}}}{\partial z}=\rho \frac{\partial^{2} w}{\partial t^{2}} .
\end{aligned}
$$


We assume that the non-homogeneities for the media $M_{1}$ and $M_{2}$ are given by

$$
\begin{aligned}
& D_{\lambda}=\sum_{K=0}^{n} \lambda_{K} e^{m z} \frac{\partial^{K}}{\partial t^{K}}, \quad D_{\mu}=\sum_{K=0}^{n} \mu_{K} e^{m z} \frac{\partial^{K}}{\partial t^{K}}, \\
& D_{\beta}=\sum_{K=0}^{n} \beta_{K} e^{m z} \frac{\partial^{K}}{\partial t^{K}}, \quad D_{m_{e}}=\sum_{K=0}^{n}\left(\mu_{e}\right)_{K} e^{m z} \frac{\partial^{K}}{\partial t^{K}}, \quad \rho=\rho_{0} \mathrm{e}^{m z},
\end{aligned}
$$

and

$$
\begin{aligned}
& D_{\lambda}^{\prime}=\sum_{K=0}^{n} \lambda_{K}^{\prime} e^{l z} \frac{\partial^{K}}{\partial t^{K}}, \quad D_{\mu}=\sum_{K=0}^{n} \mu_{K}^{\prime} e^{l z} \frac{\partial^{K}}{\partial t^{K}}, \quad D_{\beta}=\sum_{K=0}^{n} \beta_{K} e^{l z} \frac{\partial^{K}}{\partial t^{K}}, \\
& D_{\mu_{e}}^{\prime}=\sum_{K=0}^{n}\left(\mu_{e}^{\prime}\right)_{K} e^{m z} \frac{\partial^{K}}{\partial t^{K}}, \quad \rho^{\prime}=\rho_{0}^{\prime} e^{l z}
\end{aligned}
$$

where $\lambda_{0}, M_{0}, \lambda_{0}^{\prime}, \mu_{0}^{\prime}$ are elastic constants, whereas $\beta_{0}, \beta_{0}^{\prime}$ are thermal parameters $\rho_{0}, \rho_{0}^{\prime}, m, n$ are constants. $\lambda_{K},\left(\mu_{e}\right)_{K}(K=0,1,2, \ldots . n)$ are the parameters associated with $K$-th order visco-elasticity and $\beta_{K}$ and $\left(\mu_{e}\right)(K=1,2, \ldots ., n)$ are the thermal and magnetic parameters associated with $K$-th order. $T$ is the absolute temperature over the initial temperature $T_{0}$.

Due to a temperature rise of the material medium, it has been observed that all the parameters representing the elastic property, the effect of viscosity and thermal field depend on the temperature and ultimately depend on time $t$. In a thermo viscoelastic solid, the thermal parameters $\beta_{K}(K=0,1, \ldots \ldots n)$ are given by $\beta_{K}=\left(3 \lambda_{K}+2 \mu_{K}\right) \alpha_{t}$, where $\alpha_{t}$ is the coefficient of linear expansion of the solid.

$$
\begin{aligned}
& \left(G_{\lambda}+G_{\mu}+H_{0}^{2} G_{\mu_{e}}\right) \frac{\partial \Delta}{\partial x}+G_{\mu} \nabla^{2} u+m G_{\mu}\left(\frac{\partial u}{\partial z}+\frac{\partial w}{\partial x}\right)-G_{\beta} \frac{\partial T}{\partial x}=\rho_{0} \frac{\partial^{2} u}{\partial t^{2}}, \\
& G_{\mu} \nabla^{2} v+m G_{\mu} \frac{\partial v}{\partial z}=\rho_{0} \frac{\partial^{2} v}{\partial t^{2}} \\
& \left(G_{\lambda}+G_{\mu} H_{0}^{2} G_{\mu_{e}}\right) \frac{\partial \Delta}{\partial z}+G_{\mu} \nabla^{2} w+\Delta G_{\lambda} m+2 G_{\mu} m \frac{\partial w}{\partial z}-G_{\beta} \frac{\partial T}{\partial z}+ \\
& -m G_{B} T m H_{0}^{2} D G_{\mu_{e}}=\rho_{0} \frac{\partial^{2} w}{\partial t^{2}}
\end{aligned}
$$

where,

$$
\begin{gathered}
G_{\lambda}=\sum_{K=0}^{n} \lambda_{K} \frac{\partial^{K}}{\partial t^{K}}, \quad G_{\mu}=\sum_{K=0}^{n} \mu_{K} \frac{\partial^{K}}{\partial t^{K}}, \quad G_{\beta}=\sum_{K=0}^{n} \beta_{K} \frac{\partial^{K}}{\partial t^{K}}, \\
G_{\mu_{e}}=\sum_{K=0}^{n}\left(\mu_{e}\right)_{K} \frac{\partial^{K}}{\partial t^{K}}, \quad \nabla^{2}=\frac{\partial^{2}}{\partial x^{2}}+\frac{\partial^{2}}{\partial z^{2}} .
\end{gathered}
$$


To investigate the surface wave propagation along the direction of $O x$, we introduce the displacement potential $\phi(x, z, t)$ and $\psi(x, z, t)$ which are related to the displacement components as follows

$$
u=\frac{\partial \phi}{\partial x}-\frac{\partial \psi}{\partial z}, \quad w=\frac{\partial \phi}{\partial z}+\frac{\partial \psi}{\partial x}
$$

Substituting Eq.(2.8) in Eqs (2.6a), (2.6b) and (2.6c), we get

$$
\begin{aligned}
& G_{R} \nabla^{2} \phi+m G_{S}\left(2 \frac{\partial \phi}{\partial z}+\frac{\partial \psi}{\partial x}\right)-G_{L}^{T}=\frac{\partial^{2} \phi}{\partial t^{2}} \\
& G_{S} \nabla^{2} v+m G_{S} \frac{\partial v}{\partial z}=\frac{\partial^{2} v}{\partial t^{2}} \\
& \mathrm{G}_{\mathrm{S}} \nabla^{2} \psi+m G_{P} \frac{\partial \phi}{\partial x}+2 m G_{S} \frac{\partial \psi}{\partial z}=\frac{\partial^{2} \psi}{\partial t^{2}}
\end{aligned}
$$

where,

$$
\begin{array}{ll}
U_{K R}^{2}=\frac{\lambda_{K}+2 \mu_{K}+H_{0}^{2}\left(\mu_{e}\right)_{K}}{\rho_{0}}, & U_{K S}^{2}=\frac{\mu_{K}}{\rho_{0}}, \\
U_{K P}^{2}=\frac{\lambda_{K}+H_{0}^{2}\left(\mu_{e}\right)_{K}}{\rho_{0}}, & U_{K L}^{2}=\frac{\beta_{K}}{\rho_{0}},
\end{array}
$$

and

$$
\begin{array}{rlrl}
G_{R} & =\sum_{K=0}^{n} U_{K R}^{2} \frac{\partial^{K}}{\partial t^{K}}, & G_{S} & =\sum_{K=0}^{n} U_{K S}^{2} \frac{\partial^{K}}{\partial t^{K}}, \\
G_{P}=\sum_{K=0}^{n} U_{K P}^{2} \frac{\partial^{K}}{\partial t^{K}}, & G_{L}=\sum_{K=0}^{n} U_{K L}^{2} \frac{\partial^{K}}{\partial t^{K}} .
\end{array}
$$

To determine $T$, Fourier's law of heat conduction is used

$$
p \nabla^{2} T=C_{v} \frac{\partial T}{\partial t}+T_{0} G_{L} \frac{\partial}{\partial t}\left(\nabla^{2} \phi\right)
$$

where $K$ is the thermal conductivity and obeys the law as given by $K=K_{0} e^{m z}, p=\frac{K_{0}}{\rho_{0}}$ and $C_{v}$ is the specific heat of the body at constant volume.

Further, similar relations in the medium $M_{2}$ can be found out by replacing $\lambda_{K}, \mu_{K}, \beta_{K}, \rho_{0}$ by $\lambda_{K}^{\prime}, \mu_{K}^{\prime}, \beta_{K}^{\prime}, \rho_{0}^{\prime}$ and so on. 


\section{Solution of the problem}

Now our main objectives to solve Eqs (2.9a), (2.9b), (2.9c) and Eq.(2.11). We seek the solutions in the following forms

$$
(\phi, \psi, T, v)=\left[f(z), g(z), T_{l}(z), h(z)\right] e^{i \alpha(x-c t)} .
$$

Using Eq.(3.1) in Eqs (2.9a), (2.9b), (2.9c) and Eq.(2.11), we get a set of differential equations for the medium $M_{1}$ as follows

$$
\begin{aligned}
& \frac{d^{2} f}{d z^{2}}+2 m f_{1}^{2} \frac{d f}{d z}+h_{1}^{2} f+i \alpha m f_{1}^{2} g-g_{1}^{2} T_{1}=0 \\
& \frac{d^{2} h}{d z^{2}}+m \frac{d h}{d z}+K_{l}^{2} h=0 \\
& \frac{d^{2} g}{d z^{2}}+2 m \frac{d g}{d z}+K_{l}^{2} g+i \alpha m l_{1}^{2} f=0 \\
& \frac{d^{2} T_{1}}{d z^{2}}+A T_{1}+B\left(\frac{d^{2} f}{d z^{2}}-\alpha^{2} f\right)=0
\end{aligned}
$$

where,

$$
\begin{array}{ll}
f_{l}^{2}=\frac{\sum_{K=0}^{n} U_{K S}^{2}(-i \alpha c)^{K}}{\sum_{K=0}^{n} U_{K R}^{2}(-i \alpha c)^{K}}, & h_{l}^{2}=\frac{\alpha^{2} c^{2}}{\sum_{K=0}^{n} U_{K R}^{2}(-i \alpha c)^{K}}-\alpha^{2}, \\
K_{1}^{2}=\frac{\alpha^{2} c^{2}}{\sum_{K=0}^{n} U_{K S}^{2}(-i \alpha c)^{K}}-\alpha^{2}, & \\
l_{I}^{2}=\frac{\sum_{K=0}^{n} U_{K P}^{2}(-i \alpha c)^{K}}{\sum_{K=0}^{n} U_{K S}^{2}(-i \alpha c)^{K}}, & g_{1}^{2}=\frac{\sum_{K=0}^{n} U_{K L}^{2}(-i \alpha c)^{K}}{\sum_{K=0}^{n} U_{K R}^{2}(-i \alpha c)^{K}}, \\
A=\frac{C_{v} i \alpha c}{p}-\alpha^{2}, & B=\frac{i \alpha c T_{0}}{p}-G_{L},
\end{array}
$$


and those for the medium $M_{2}$ are given by

$$
\begin{aligned}
& \frac{d^{2} f}{d z^{2}}+2 l l_{1}^{\prime 2} \frac{d f}{d z}+h_{l}^{\prime 2} f+i \alpha l f_{1}^{\prime 2} g-g_{l}^{\prime 2} T_{I}=0 \\
& \frac{d^{2} h}{d z^{2}}+l \frac{d h}{d z}+K_{l}^{\prime 2} h=0 \\
& \frac{d^{2} g}{d z^{2}}+2 l \frac{d g}{d z}+K_{l}^{\prime 2} g+i \alpha l \cdot l_{1}^{\prime 2} f=0 \\
& \frac{d^{2} T_{1}}{d z^{2}}+A^{\prime} T_{l}+B^{\prime}\left(\frac{d^{2} f}{d z^{2}}-\alpha^{2} f\right)=0
\end{aligned}
$$

where,

$$
\begin{aligned}
& f_{1}^{\prime 2}=\frac{\sum_{K=0}^{n} U_{K S}^{\prime 2}(-i \alpha c)^{K}}{\sum_{K=0}^{n} U_{K R}^{\prime 2}(-i \alpha c)^{K}}, \quad h_{1}^{\prime 2}=\frac{\alpha^{2} c^{2}}{\sum_{K=0}^{n} U_{K R}^{\prime 2}(-i \alpha c)^{K}}-\alpha^{2}, \\
& K_{l}^{\prime 2}=\frac{\alpha^{2} c^{2}}{\sum_{K=0}^{n} U_{K S}^{\prime 2}(-i \alpha c)^{K}}-\alpha^{2}, \\
& l_{1}^{\prime 2}=\frac{\sum_{K=0}^{n} U_{K P}^{\prime 2}(-i \alpha c)^{K}}{\sum_{K=0}^{n} U_{K S}^{\prime 2}(-i \alpha c)^{K}}, \quad g_{1}^{\prime 2}=\frac{\sum_{K=0}^{n} U_{K L}^{\prime 2}(-i \alpha c)^{K}}{\sum_{K=0}^{n} U_{K R}^{\prime 2}(-i \alpha c)^{K}}, \quad B^{\prime}=\frac{i \alpha c T_{0}}{p^{\prime}}-G_{L}^{\prime} .
\end{aligned}
$$

Equations (3.2) and (3.4) must have exponential solutions so that $f, g, T_{1}, h$ will describe surface waves, and they must become vanishing small as $z \rightarrow \infty$.

Hence for the medium $M_{1}$

$$
\begin{aligned}
& \phi(x, z, t)=\left\{A_{1} e^{-\lambda_{1} z}+B_{1} e^{-\lambda_{2} z}+C_{1} e^{-\lambda_{3} z}\right\} e^{i \alpha(x-c t)}, \\
& \psi(x, z, t)=\left\{A_{2} e^{-\lambda_{1} z}+B_{2} e^{-\lambda_{2} z}+C_{2} e^{-\lambda_{3} z}\right\} e^{i \alpha(x-c t)}, \\
& T(x, z, t)=\left\{A_{3} e^{-\lambda_{1} z}+B_{3} e^{-\lambda_{2} z}+C_{3} e^{-\lambda_{3} z}\right\} e^{i \alpha(x-c t)}, \\
& v(x, z, t)=C e^{-\lambda_{4} z+i \alpha(x-c t)},
\end{aligned}
$$


and similarly for the medium $M_{2}$

$$
\begin{aligned}
& \phi(x, z, t)=\left\{A_{1}^{\prime} e^{-\lambda_{1}^{\prime} z}+B_{1}^{\prime} e^{-\lambda_{2}^{\prime} z}+C_{1}^{\prime} e^{-\lambda_{3}^{\prime} z}\right\} e^{i \alpha(x-c t)}, \\
& \psi(x, z, t)=\left\{A_{2}^{\prime} e^{-\lambda_{1}^{\prime} z}+B_{2}^{\prime} e^{-\lambda_{2}^{\prime} z}+C_{2}^{\prime} e^{-\lambda_{3}^{\prime} z}\right\} e^{i \alpha(x-c t)}, \\
& T(x, z, t)=\left\{A_{3}^{\prime} e^{-\lambda_{1}^{\prime} z}+B_{3}^{\prime} e^{-\lambda_{2}^{\prime} z}+C_{3}^{\prime} e^{-\lambda_{3}^{\prime} z}\right\} e^{i \alpha(x-c t)}, \\
& v(x, z, t)=C^{\prime} e^{-\lambda_{4}^{\prime} z+i \alpha(x-c t)}
\end{aligned}
$$

where $\lambda_{j}$ and $\lambda_{j}^{\prime}(j=1,2,3)$ are the real roots of the equation

$$
\lambda^{6}+\xi_{1} \lambda^{5}+\xi_{2} \lambda^{4}+\xi_{3} \lambda^{3}+\xi_{4} \lambda^{2}+\xi_{5} \lambda+\xi_{6}=0
$$

where,

$$
\begin{aligned}
& \xi_{1}=2 m\left\{1+f_{1}^{2}\right\}, \quad \xi_{2}=K_{1}^{2}+A+4 m^{2}+h_{1}^{2}+B g_{1}^{2}, \\
& \xi_{3}=2 m A+2 f_{1}^{2} m\left(K_{l}^{2}+A\right)+2 m h_{l}^{2}+2 m B g_{1}^{2}, \\
& \xi_{4}=A K_{1}^{2}+4 m^{2} A f_{1}^{2}+\left(K_{l}^{2}+A\right) h_{1}^{2}+\alpha^{2} m^{2} l_{1}^{2} f_{l}^{2}+B K_{l}^{2} g_{1}^{2}-\alpha^{2} B g_{1}^{2} \text {, } \\
& \xi_{5}=2 m A K_{1}^{2} f_{1}^{2}+2 m A h_{1}^{2}-2 m \alpha^{2} B g_{1}^{2}, \\
& \xi_{6}=A K_{l}^{2} h_{l}^{2}+A \alpha^{2} m^{2} l_{l}^{2} f_{l}^{2}-\alpha^{2} B K_{l}^{2} g_{l}^{2}, \\
& \lambda^{\prime 6}+\xi_{1}^{\prime} \lambda^{\prime 5}+\xi_{2}^{\prime} \lambda^{\prime 4}+\xi_{3}^{\prime} \lambda^{\prime 3}+\xi_{4}^{\prime} \lambda^{\prime 2}+\xi_{5}^{\prime} \lambda^{\prime}+\xi_{6}^{\prime}=0
\end{aligned}
$$

where,

$$
\begin{aligned}
& \xi_{l}^{\prime}=2 l\left\{1+f_{l}^{\prime 2}\right\}, \quad \xi_{2}^{\prime}=K_{l}^{\prime 2}+A^{\prime}+4 l^{2}+h_{l}^{\prime 2}+B^{\prime} g_{l}^{\prime 2}, \\
& \xi_{3}^{\prime}=2 l A^{\prime}+2 l f_{l}^{\prime 2}\left(K_{l}^{\prime 2}+A\right)+2 l h_{l}^{\prime 2}+2 l B^{\prime} g_{l}^{\prime 2}, \\
& \xi_{4}^{\prime}=A^{\prime} K_{l}^{\prime 2}+4 l^{2} A^{\prime} f_{l}^{\prime 2}+\left(K_{l}^{\prime 2}+A^{\prime}\right) h_{l}^{\prime 2}+\alpha^{2} l^{2} l_{l}^{\prime 2} f_{l}^{\prime 2}+B^{\prime} K_{l}^{\prime 2} g_{l}^{\prime 2}-\alpha^{2} B^{\prime} g_{l}^{\prime 2}, \\
& \xi_{5}^{\prime}=2 l A^{\prime} K_{l}^{\prime 2} f_{l}^{\prime 2}+2 l A^{\prime} h_{l}^{\prime 2}-2 l \alpha^{2} B^{\prime} g_{l}^{\prime 2} \\
& \xi_{6}^{\prime}=A^{\prime} K_{l}^{\prime 2} h_{l}^{\prime 2}+A^{\prime} \alpha^{2} l^{2} l_{l}^{\prime 2} f_{l}^{\prime 2}-\alpha^{2} B^{\prime} K_{l}^{\prime 2} g_{l}^{\prime 2} .
\end{aligned}
$$


and

$$
\lambda_{4}, \lambda_{4}^{\prime}=\left\{m+\left(m^{2}-4 K_{l}^{2}\right)^{1 / 2}\right\} / 2, \quad 1+\left(1^{2}-4 K_{l}^{\prime 2}\right)^{1 / 2} / 2
$$

where the symbols used in Eqs (3.8) and (3.10) are given by Eqs (3.3) and (3.5).

The constants $A_{j}, B_{j}, C_{j}(j=1,2,3)$ are related with $A_{j}^{\prime}, B_{j}^{\prime}, C_{j}^{\prime}(j=1,2,3)$ in Eqs (3.6a) and (3.6b) by means of first equations in Eqs (3.2) and (3.4).

Equating the coefficients of $e^{-\lambda_{1} z}, e^{-\lambda_{2} z}, e^{-\lambda_{3} z}, e^{-\lambda_{1}^{\prime} z}, e^{-\lambda_{2}^{\prime} z}, e^{-\lambda_{3}^{\prime} z}$ to zero, after substituting Eqs (3.6a) and (3.6b) in the first and 3rd equations of Eq.(3.2) and Eq.(3.4), respectively, we get

$$
A_{2}=\gamma_{1} A_{1}, \quad B_{2}=\gamma_{2} B_{1}, \quad C_{2}=\gamma_{3} C_{1},
$$

and

$$
A_{3}=\delta_{1} A_{1}, \quad B_{3}=\delta_{2} B_{1}, \quad C_{3}=\delta_{3} C_{1}
$$

where,

$$
\begin{aligned}
& \gamma_{j}=\frac{-i \alpha m l_{1}^{2}}{\lambda_{j}^{2}-2 m \lambda_{j}+K_{l}^{2}}, \quad j=(1,2,3), \\
& \delta_{j}=\frac{1}{g_{l}^{2}}\left[\lambda_{j}^{2}-2 m f_{l}^{2} \lambda_{j}+h_{l}^{2}+i \alpha m f_{l}^{2} \gamma_{j}\right], \quad j=1,2,3 .
\end{aligned}
$$

A similar result holds for the medium $M_{2}$ and usual symbols are replaced by dashes respectively.

\section{Boundary conditions}

(i) The displacement components, temperature and temperature flux at the boundary surface between the media $M_{1}$ and $M_{2}$ must be continuous at all times and positions

i.e., $\quad\left[u, v, w, T, p \frac{\partial T}{\partial z}\right]_{M_{1}}=\left[u, v, w, T, p^{\prime} \frac{\partial T}{\partial z}\right]_{M_{2}}$.

(ii) The stress components $\tau_{31}, \tau_{32}, \tau_{33}$ must be continuous at the boundary $z=0$,

i.e., $\quad\left[\tau_{31}, \tau_{32}, \tau_{33}\right]_{M_{1}}=\left[\tau_{31}, \tau_{32}, \tau_{33}\right]_{M_{2}} \quad$ at $\quad z=0, \quad$ respectively

where,

$$
\begin{aligned}
& \tau_{31}=D_{\mu}\left(2 \frac{\partial^{2} \phi}{\partial x \partial z}+\frac{\partial^{2} \psi}{\partial x^{2}}-\frac{\partial^{2} \psi}{\partial z^{2}}\right), \\
& \tau_{32}=D_{\mu} \frac{\partial v}{\partial z}, \\
& \tau_{33}=D_{\lambda} \nabla^{2} \phi+2 D_{\mu}\left(\frac{\partial^{2} \phi}{\partial z^{2}}+\frac{\partial^{2} \phi}{\partial x \partial z}\right)-D_{B} T+D_{m e} H_{0}^{2} \nabla^{2} \phi .
\end{aligned}
$$


Applying the boundary conditions, we get

$$
\begin{aligned}
& A_{1}\left(1-i \gamma_{1} \zeta_{1}\right)+B_{1}\left(1-i \gamma_{2} \zeta_{2}\right)+C_{1}\left(1-i \gamma_{3} \zeta_{3}\right)-A_{1}^{\prime}\left(1-i^{\prime} \gamma_{3} \zeta_{1}^{\prime}\right)+ \\
& -B_{1}\left(1-i \gamma_{2}^{\prime} \zeta_{2}^{\prime}\right)-C_{1}^{\prime}\left(1-i \gamma_{3}^{\prime} \zeta_{3}^{\prime}\right)=0 \text {, } \\
& C=C^{\prime}, \\
& A_{1}\left(\gamma_{1}+i \zeta_{1}\right)+B_{1}\left(\gamma_{2}+i \zeta_{2}\right)+C_{1}\left(\gamma_{3}+i \zeta_{3}\right)-A_{1}^{\prime}\left(\gamma_{1}^{\prime}+i \zeta_{1}^{\prime}\right)+ \\
& -B_{1}^{\prime}\left(\gamma_{2}^{\prime}+i \zeta_{2}^{\prime}\right)-C_{1}^{\prime}\left(\gamma_{3}^{\prime}+i \zeta_{3}^{\prime}\right)=0 \text {, } \\
& \delta_{1} A_{1}+\delta_{2} B_{1}+\delta_{3} C_{1}=\delta_{1}^{\prime} A_{1}^{\prime}+\delta_{2}^{\prime} B_{1}^{\prime}+\delta_{3}^{\prime} C_{1}^{\prime}, \\
& p \lambda_{1} \delta_{1} A_{1}+p \lambda_{2} \delta_{2} B_{2}+p \lambda_{3} \delta_{3} C_{1}-p^{\prime} \lambda_{1}^{\prime} \delta_{1}^{\prime} A_{1}^{\prime}+p^{\prime} \lambda_{2}^{\prime} \delta_{2}^{\prime} B_{1}^{\prime}-p^{\prime} \lambda_{3}^{\prime} \delta_{3}^{\prime} C_{1}^{\prime}=0, \\
& \mu_{K}^{*}\left[\left(2 i \zeta_{1}+\gamma_{1}+\zeta_{1}^{2} \gamma_{1}\right) A_{1}+\left(2 i \zeta_{2}+\gamma_{2}+\zeta_{2}^{2} \lambda_{2}\right) B_{1}+\left(2 i \zeta_{3}+\gamma_{3}+\zeta_{3}^{2} \gamma_{3}\right) C_{1}\right]= \\
& =\mu_{K}^{\prime *}\left[\left(2 i \zeta_{1}^{\prime}+\gamma_{1}^{\prime}+\zeta_{1}^{\prime 2} \gamma_{1}^{\prime}\right) A_{1}^{\prime}+\left(2 i \zeta_{2}^{\prime}+\gamma_{2}^{\prime}+\zeta_{2}^{\prime 2} \gamma_{2}^{\prime}\right) B_{1}^{\prime}+\left(2 i \zeta_{3}^{\prime}+\gamma_{3}^{\prime}+\zeta_{3}^{\prime 2} \gamma_{3}^{\prime}\right) C_{1}^{\prime}\right] \text {, } \\
& \mu_{K}^{*}\left[-\lambda_{4} C\right]=\mu_{K}^{\prime *}\left[-\lambda_{4}^{\prime} C^{\prime}\right] \\
& A_{1}\left[\left(\lambda_{K}^{*}+\left(\mu_{e}\right)_{K}^{*} H_{0}^{2}\right)\left(\zeta_{1}^{2}-1\right)+2 \mu_{K}^{*}\left(\zeta_{1}^{2}-i \zeta_{1}\right)-\beta_{K}^{*} \delta_{l}\right]+ \\
& +B_{1}\left[\left(\lambda_{K}^{*}+\left(\mu_{e}\right)_{K}^{*} H_{0}^{2}\right)\left(\zeta_{2}^{2}-1\right)+2 \mu_{K}^{*}\left(\zeta_{2}^{2}-i \zeta_{2}\right)-\beta_{K}^{*} \delta_{2}\right]+ \\
& +C_{1}\left[\left(\lambda_{K}^{*}+\left(\mu_{e}\right)_{K}^{*} H_{0}^{2}\right)\left(\zeta_{3}^{2}-1\right)+2 \mu_{K}^{*}\left(\zeta_{3}^{2}-i \zeta_{3}\right)-\beta_{K}^{*} \delta_{3}\right]= \\
& =A_{I}^{\prime}\left[\left(\lambda_{K}^{\prime *}+\left(\mu_{e}^{\prime}\right)_{K}^{*} H_{0}^{2}\right)\left(\zeta_{l}^{\prime 2}-1\right)+2 \mu_{K}^{\prime *}\left(\zeta_{1}^{\prime 2}-i \zeta_{l}^{\prime}\right)-\beta_{K}^{\prime *} \delta_{I}^{\prime}\right]+ \\
& B_{1}^{\prime}\left[\left(\lambda_{K}^{\prime *}+\left(\mu_{e}^{\prime}\right)_{K}^{*} H_{0}^{2}\right)\left(\zeta_{2}^{\prime 2}-1\right)+2 \mu_{K}^{\prime *}\left(\zeta_{2}^{\prime 2}-i \zeta_{2}^{\prime}\right)-\beta_{K}^{\prime *} \delta_{2}^{\prime}\right]+ \\
& C_{1}^{\prime}\left[\left(\lambda_{K}^{\prime *}+\left(\mu_{e}^{\prime}\right)_{K}^{*} H_{0}^{2}\right)\left(\zeta_{3}^{\prime 2}-1\right)+2 \mu_{K}^{\prime *}\left(\zeta_{3}^{\prime 2}-i \zeta_{3}^{\prime}\right)-\beta_{K}^{\prime *} \delta_{3}^{\prime}\right]
\end{aligned}
$$

where,

$$
\zeta_{j}=\frac{\lambda_{j}}{\alpha}, \quad \zeta_{j}^{\prime}=\frac{\lambda_{j}^{\prime}}{\alpha}, \quad j=1,2,3
$$

and

$$
\begin{array}{ll}
\lambda_{K}^{*}=\sum_{K=0}^{n} \lambda_{K}(-i \alpha c)^{K}, & \mu_{\mathrm{K}}^{*}=\sum_{K=0}^{n} \mu_{K}(-i \alpha c)^{K}, \\
\beta_{\mathrm{K}}^{*}=\sum_{K=0}^{n} \beta_{K}(-i \alpha c)^{K}, & \left(\mu_{e}\right)_{K}^{*}=\sum_{K=0}^{n}\left(\mu_{e}\right)_{K}(-i \alpha c)^{K},
\end{array}
$$




$$
\begin{array}{ll}
\lambda_{K}^{\prime *}=\sum_{K=0}^{n} \lambda_{K}^{\prime}(-i \alpha c)^{K}, & \mu_{K}^{\prime *}=\sum_{K=0}^{n} \mu_{K}^{\prime}(-i \alpha c)^{K}, \\
\beta_{K}^{\prime *}=\sum_{K=0}^{n} \beta_{K}^{\prime}(-i \alpha c)^{K}, & \left(\mu_{e}^{\prime}\right)_{K}^{*}=\sum_{K=0}^{n}\left(\mu_{e}^{\prime}\right)_{K}(-i \alpha c)^{K} .
\end{array}
$$

From Eqs (4.2b) and (4.2g), we have $C=C^{\prime}=0$. Thus there is no propagation of displacement $v$. Hence $\mathrm{SH}$-waves do not occur in this case.

Finally, eliminating the constants $A_{1}, B_{1}, C_{1}, A^{\prime}{ }_{1}, B_{1}{ }_{1}, C^{\prime}{ }_{1}$, from the remaining equations, we get

$$
\operatorname{det}\left(a_{i j}\right)=0, \quad i, j=1,2,3,4,5,6
$$

where,

$$
\begin{aligned}
& a_{11}=1-i \gamma_{1} \zeta_{1}, \quad a_{12}=1-i \gamma_{2} \zeta_{2}, \quad a_{13}=1-i \gamma_{3} \zeta_{3}, \\
& a_{14}=\left(i \gamma_{1}^{\prime} \zeta_{1}^{\prime}-1\right), \quad a_{15}=\left(i \gamma_{2}^{\prime} \zeta_{2}^{\prime}-1\right), \quad a_{16}=\left(i \gamma_{3}^{\prime} \zeta_{3}^{\prime}-1\right), \\
& a_{21}=\gamma_{1}+i \zeta_{1}, \quad a_{22}=\gamma_{2}+i \zeta_{2}, \quad a_{23}=\gamma_{3}+i \zeta_{3}, \\
& a_{24}=\left(\gamma_{1}^{\prime}+i \zeta_{1}^{\prime}\right), \quad a_{25}=\left(\gamma_{2}^{\prime}+i \zeta_{2}^{\prime}\right), \quad a_{26}=\left(\gamma_{3}^{\prime}+i \zeta_{3}^{\prime}\right), \\
& a_{31}=\delta_{1}, \quad a_{32}=\delta_{2}, \quad a_{33}=\delta_{3}, \quad a_{34}=-\delta_{1}^{\prime}, \quad a_{35}=-\delta_{2}^{\prime}, \\
& a_{36}=-\delta_{3}^{\prime}, \quad a_{41}=p \lambda_{1} \delta_{1}, \quad a_{42}=p \lambda_{2} \delta_{2}, \quad a_{43}=p \lambda_{3} \delta_{3}, \\
& a_{44}=-p^{\prime} \lambda_{1}^{\prime} \delta_{1}^{\prime}, \quad a_{45}=-p^{\prime} \lambda_{2}^{\prime} \delta_{2}^{\prime}, \quad a_{46}=-p^{\prime} \lambda_{3}^{\prime} \delta_{3}^{\prime}, \\
& a_{51}=\mu_{K}^{*}\left(2 i \zeta_{1}+\gamma_{1}+\gamma_{1} \zeta_{1}^{2}\right), \quad a_{52}=\mu_{K}^{*}\left(2 i \zeta_{2}+\gamma_{2}+\gamma_{2} \zeta_{2}^{2}\right), \\
& a_{53}=\mu_{K}^{*}\left(2 i \zeta_{3}+\gamma_{3}+\gamma_{3} \zeta_{3}^{2}\right), \quad a_{54}=\mu_{K}^{*}\left(2 i \zeta_{1}^{\prime}+\gamma_{1}^{\prime}+\gamma_{1}^{\prime} \zeta_{1}^{\prime 2}\right), \\
& a_{55}=\mu_{K}^{\prime *}\left(2 i \zeta_{2}^{\prime}+\gamma_{2}^{\prime}+\gamma_{1}^{\prime} \zeta_{2}^{\prime 2}\right), \quad a_{56}=\mu_{K}^{\prime *}\left(2 i \zeta_{3}^{\prime}+\gamma_{3}^{\prime}+\gamma_{3}^{\prime} \zeta_{3}^{\prime 2}\right), \\
& a_{61}=\left(\lambda_{K}^{*}+\left(\mu_{e}\right)_{K}^{*} H_{0}^{2}\right)\left(\zeta_{1}^{2}-1\right)+2 \mu_{K}^{*}\left(\zeta_{1}^{2}-i \zeta_{1}\right)-\beta_{K}^{*} \delta_{1}, \\
& a_{62}=\left(\lambda_{K}^{*}+\left(\mu_{e}\right)_{K}^{*} H_{0}^{2}\right)\left(\zeta_{2}^{2}-1\right)+2 \mu_{K}^{*}\left(\zeta_{2}^{2}-i \zeta_{2}\right)-\beta_{K}^{*} \delta_{2}, \\
& a_{63}=\left(\lambda_{K}^{*}+\left(\mu_{e}\right)_{K}^{*} H_{0}^{2}\right)\left(\zeta_{3}^{2}-1\right)+2 \mu_{K}^{*}\left(\zeta_{3}^{2}-i \zeta_{3}\right)-\beta_{K}^{*} \delta_{3},
\end{aligned}
$$




$$
\begin{aligned}
& a_{64}=\left(\lambda_{K}^{\prime *}+\left(\mu_{e}^{\prime}\right)_{K}^{*} H_{0}^{2}\right)\left(\zeta_{1}^{\prime 2}-1\right)+2 \mu_{K}^{\prime *}\left(\zeta_{1}^{\prime 2}-i \zeta_{1}^{\prime}\right)-\beta_{K}^{\prime *} \delta_{1}^{\prime}, \\
& a_{65}=\left(\lambda_{K}^{\prime *}+\left(\mu_{e}^{\prime}\right)_{K}^{*} H_{0}^{2}\right)\left(\zeta_{2}^{\prime 2}-1\right)+2 \mu_{K}^{\prime *}\left(\zeta_{2}^{\prime 2}-i \zeta_{2}^{\prime}\right)-\beta_{K}^{\prime *} \delta_{2}^{\prime}, \\
& a_{66}=\left(\lambda_{K}^{\prime *}+\left(\mu_{e}^{\prime}\right)_{K}^{*} H_{0}^{2}\right)\left(\zeta_{3}^{\prime 2}-1\right)+2 \mu_{K}^{\prime *}\left(\zeta_{3}^{\prime 2}-i \zeta_{3}^{\prime}\right)-\beta_{K}^{\prime *} \delta_{3}^{\prime} .
\end{aligned}
$$

From Eq.(4.3), we obtain the velocity of surface waves in a common boundary between two viscoelastic, non-homogeneous solid media under the influence of the thermal and magnetic field, where the viscosity is of general $n$-th order involving the time rate of change of strain.

\section{Particular cases}

\section{Stoneley waves:}

They are a generalised form of Rayleigh waves in which we assume that waves are propagated along the common boundary of two semi-infinite media $M_{1}$ and $M_{2}$. Thus Eq.(4.3) determines the wave velocity equation for Stoneley waves in the case of general magneto-thermo viscoelastic, non-homogeneous solid media of $n$-th order involving the time rate of strain. Clearly, from Eq.(4.3) it is follows that the wave velocity equation for Stoneley waves depends upon the non-homogeneity of the material medium, temperature, magnetic and viscous field. This equation, of course, is in good agreement with the corresponding classical result, when the effects of the thermal, magnetic and viscous field and nonhomogeneity are absent.

\section{Rayleigh waves:}

To investigate the possibility of Rayleigh waves in thermo viscoelastic, non-homogeneous elastic media, we replace media $M_{2}$ by vacuum, in the proceeding problem. We also note the SH-waves do not occur in this case.

Since the temperature difference across the boundary is always small, the thermal condition is given by

$$
\frac{\partial T}{\partial z}+h T=0 \quad \text { at } \quad z=0, \quad \text { respectively }
$$

Thus Eqs (4.2f) and (4.2h) reduce to,

$$
\begin{aligned}
& \left(2 i \zeta_{1}+\gamma_{1}+\gamma_{1} \zeta_{1}^{2}\right) A_{1}+\left(2 i \zeta_{2}+\gamma_{2}+\gamma_{2} \zeta_{2}^{2}\right) B_{1}+\left(2 i \zeta_{3}+\gamma_{3}+\gamma_{3} \zeta_{3}^{2}\right) C_{1}=0, \\
& {\left[\left(\lambda_{K}^{*}+\left(\mu_{e}\right)_{K}^{*} H_{0}^{2}\right)\left(\zeta_{1}^{2}-1\right)+2 \mu_{K}^{*}\left(\zeta_{1}^{2}-i \zeta_{1}\right)-\beta_{K}^{*} \delta_{1}\right] A_{1}+} \\
& +\left[\left(\lambda_{K}^{*}+\left(\mu_{e}\right)_{K}^{*} H_{0}^{2}\right)\left(\zeta_{2}^{2}-1\right)+2 \mu_{K}^{*}\left(\zeta_{2}^{2}-i \zeta_{2}\right)-\beta_{K}^{*} \delta_{2}\right] B_{1}+ \\
& +\left[\left(\lambda_{K}^{*}+\left(\mu_{e}\right)_{K}^{*} H_{0}^{2}\right)\left(\zeta_{3}^{2}-1\right)+2 \mu_{K}^{*}\left(\zeta_{3}^{2}-i \zeta_{3}\right)-\beta_{K}^{*} \delta_{3}\right] C_{1}=0 .
\end{aligned}
$$


From Eq.(5.1), we have

$$
\left(\lambda_{1}-h\right) \delta_{1} A_{1}+\left(\lambda_{2}-h\right) \delta_{2} B_{1}+\left(\lambda_{3}-h\right) \delta_{3} C_{1}=0 .
$$

Eliminating $A_{1}, B_{1}$ and $C_{1}$ from Eqs (5.2a), (5.2b) and (5.2c), we get

$$
\operatorname{det}\left(b_{i j}\right)=0, \quad i, j=1,2,3
$$

where,

$$
\begin{aligned}
& b_{11}=\left(2 i \zeta_{1}+\gamma_{1}+\gamma_{1} \zeta_{1}^{2}\right), \quad b_{12}=\left(2 i \zeta_{2}+\gamma_{2}+\gamma_{2} \zeta_{2}^{2}\right), \quad b_{13}=\left(2 i \zeta_{3}+\gamma_{3}+\gamma_{3} \zeta_{3}^{2}\right), \\
& b_{21}=\left[\left(\lambda_{K}^{*}+\left(\mu_{e}\right)_{K}^{*} H_{0}^{2}\right)\left(\zeta_{1}^{2}-1\right)+2 \mu_{K}^{*}\left(\zeta_{1}^{2}-i \zeta_{1}\right)-\beta_{K}^{*} \delta_{1}\right], \\
& b_{22}=\left[\left(\lambda_{K}^{*}+\left(\mu_{e}\right)_{K}^{*} H_{0}^{2}\right)\left(\zeta_{2}^{2}-1\right)+2 \mu_{K}^{*}\left(\zeta_{2}^{2}-i \zeta_{2}\right)-\beta_{K}^{*} \delta_{2}\right], \\
& b_{23}=\left[\left(\lambda_{K}^{*}+\left(\mu_{e}\right)_{K}^{*} H_{0}^{2}\right)\left(\zeta_{3}^{2}-1\right)+2 \mu_{K}^{*}\left(\zeta_{3}^{2}-i \zeta_{1}\right)-\beta_{K}^{*} \delta_{3}\right], \\
& b_{31}=\left(\lambda_{1}-h\right) \delta_{1}, \quad b_{32}=\left(\lambda_{2}-h\right) \delta_{2}, \quad b_{33}=\left(\lambda_{3}-h\right) \delta_{3} .
\end{aligned}
$$

Thus Eq.(5.3), gives the wave velocity equation for the Rayleigh waves in non-homogeneous, magneto-thermo viscoelastic solid media of $n$-th order involving time rate of strain.

From Eq.(5.3), it follows that the dispersion equation of Rayleigh waves depends upon the nonhomogeneity, the viscous, magnetic and thermal fields.

This equation, of course, is in complete agreement with the corresponding classical result by Bullen, when the effects of the thermal, magnetic viscous field and non-homogeneity are absent.

\section{Love waves:}

To investigate the possibility of Love waves in non-homogeneous, viscoelastic solid media, we introduce medium $M_{2}$ which is obtained by two horizontal plane surfaces at a distance $H$-apart, while $M_{1}$ remains infinite.

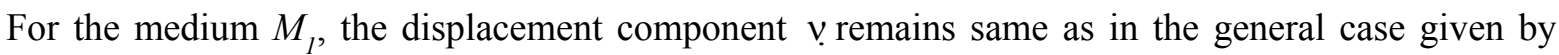
Eq.(3.6).

For the medium $M_{2}$, we preserve the full solution, since the displacement component along the $y$-axis i.e., $v$ no longer diminishes with increasing distance from the boundary surface of the two media.

Thus

$$
v^{\prime}=C_{1} e^{\lambda_{4}^{\prime} z+i \alpha(x-c t)}+C_{2} e^{-\lambda_{4}^{\prime} z+i \alpha(x-c t)}
$$

In this case, the boundary conditions are

(i) $\quad v$ and $\tau_{32}$ are continuous at $z=0$

(ii) $\tau_{32}^{\prime}=0$ at $z=-H$. 
Applying the boundary conditions (i) and (ii) and using Eqs (3.6) and (4.2), we get

$$
\begin{aligned}
& C=C_{1}+C_{2}, \\
& -\mu_{K}^{*} \lambda_{4} C=\left(\mu_{K}^{\prime}\right) *\left[\lambda_{4}^{\prime} C_{1}-\lambda_{4}^{\prime} C_{2}\right], \\
& C_{1} e^{-\lambda_{4}^{\prime} H}-C_{2} e^{\lambda_{4}^{\prime} H}=0 .
\end{aligned}
$$

On eliminating the constants $C, C_{1}$ and $C_{2}$ from Eqs (4.3), (5.1) and (5.2), we get

$$
\tanh \left(\lambda_{4}^{\prime} H\right)=-\frac{\lambda_{4} \mu_{K}^{*}}{\lambda_{4}^{\prime}\left(\mu_{K}^{\prime}\right)^{*}}
$$

Thus Eq.(5.9) gives the wave velocity equation for Love waves in a non-homogeneous, magneto, thermo viscoelastic solid medium of $n$-th order involving the time rate of strain. Clearly, it depends upon the non-homogeneity, magnetic and viscous fields and it is independent of the thermal field.

\section{Discussion and conclusions}

The present study reveals the effects of non-homogeneity, viscous, magnetic and thermal fields in the wave velocity equations corresponding to Stoneley waves, Rayleigh waves and Love waves.

Further, it is found that viscoelastic surface waves are affected by the time rate of strain parameters. These parameters influence the wave velocity to an extent depending on the corresponding constants characterizing the magneto thermo and visco-elasticity of the material. So the results of this analysis become useful in circumstances where these effects cannot be neglected. These velocities depend upon the wave number $\alpha$ confirming that these waves are affected by non-homogeneity of the material medium. Some special cases of this study in a homogeneous medium under the influence of gravity have been discussed by several authors including Sengupta et al. and Das.

Also it is noted from Eq.(5.9) is that Love waves do not depend on temperature; these are only affected by the viscous, magnetic fields and non-homogeneity of the material medium. In the absence of all fields and non-homogeneity, the dispersion equation is in complete agreement with the corresponding classical result.

Further, for Rayleigh waves in a non-homogeneous, general magneto-thermo viscoelastic solid medium of higher order including the time rate of change of strain we find that the wave velocity equation proves that there is dispersion of waves due to the presence of non-homogeneity, temperature, magnetic field and viscosity. The results are in complete agreement with the corresponding classical results in the absence of all fields and compression.

It is noted that the wave velocity equation of Stoneley waves is very similar to the corresponding problem in the classical theory of elasticity. Here also there is dispersion of waves due to the presence of non-homogeneity, magnetic field, temperature and the viscoelastic nature of the solid. Also wave velocity equation of this generalized type of surface waves in non-homogeneous magneto, thermo viscoelastic solid media of higher order including the time rate of strain is in complete agreement with the corresponding classical result in the absence of all fields and non-homogeneity.

Further, the solution of wave velocity equation for Stoneley waves cannot be determined by easy analytical methods. However, we can apply numerical techniques to solve this determinantal equation by choosing suitable values of physical constants for both media $M_{1}$ and $M_{2}$. 


\section{Nomenclature}

$$
\begin{aligned}
e_{i j i, j=1,2,3,} & - \text { strain components } \\
\boldsymbol{H}_{0} & - \text { primary magnetic field } \\
t_{0} & - \text { absolute temperature } \\
\beta_{K}(K=1,2, \ldots ., n) & - \text { thermal parameters } \\
\lambda_{0}, \mu_{0}, \lambda_{0}^{\prime}, \mu_{0}^{\prime} & - \text { elastic constants } \\
\lambda_{K^{\prime}}, \mu_{K}(K=1,2, \ldots . n) & - \text { parameters associated with } K \text {-th order visco-elasticity } \\
\left(\mu_{e}\right)(K=1,2, \ldots ., n) & \text { - magnetic parameters } \\
\rho & - \text { density of the material medium } \\
\phi, \psi \text { and } v & - \text { associated with P waves. SV waves and SH waves } \\
\tau_{i j} i, j=1,2,3, & - \text { stress components } \\
\nabla^{2}=\frac{\partial^{2}}{\partial x^{2}}+\frac{\partial^{2}}{\partial z^{2}} & - \text { Laplacian operator }
\end{aligned}
$$

\section{References}

Abd-Alla A.M. and Ahmed S.M. (1996): Rayleigh waves in an orthotropic thermoelastic medium under gravity field and initial stress. - J. Earth, Moon Planets, vol.75, pp.185-197.

Biot M.A. (1965): Mechanics of Incremental Deformations. - J. Willy.

Bland D.R. (1960): The Theory of Linear Viscoelasticity. - London: Pergamon, Monograph on the Subject Contains Many Cases of Stress Analysis.

Bromwich T.J. (1898): On the influence of gravity on elastic waves, and, in particular, on the vibrations of an elastic globe. - Proc. London Math. Soc., vol.30. pp.98-120.

Brunelle E.J. (1973): Surface wave propagation under initial tension or compression. - Bull. Seismol. Soc. Am., vol.63, pp.1895-1899.

Bullen K.E. (1965): Theory of Seismology. - Cambridge University Press.

Chattopadhyay A., Gupta S., Sharma V.K. and Kumari P. (2010): Effect of point source and heterogeneity on the propagation of $\mathrm{SH}$ - waves. - Int. J. of Appl. Math. and Mech, vol.6(9), pp.76-89.

Chattopadhyay A., Gupta S., Sharma V.K. and Kumari P. (2010): Propagation of G-type seismic waves in viscoelastic medium. - Int. J. of Appl. Math. and Mech, vol.6(9), pp.63-75.

Datta B.K. (1986): Some observation on interactions of Rayleigh waves in an elastic solid medium with the gravity field. - Rev. Roumaine Sci. Tech. Ser. Mec. Appl., vol.31, pp.369-374.

De S.K. and Sengupta P.R. (1974): Influence of gravity on wave propagation in an elastic layer. - J. Acoust. Soc. Am., vol.55, pp.919-921.

Eringen A.C. and Sahubi E.S. (1975): Linear Theory of Elastodynamics. - New York: London Academic Press, vol.2.

Ewing W.M., Jardetzky W.S. and Press F. (1957): Elastic Waves in Layered Media. - New York: McGraw-Hill.

Flugge W. (1967): Viscoelasticity. - London: Blaisdel.

Goda M.A. (1992): The effect of inhomogeneity and anisotropy on Stoneley waves. - Acta Mech., vol.93, No.1-4, pp.89-98.

Haskell N.A. (1953): The dispersion of surface waves in multilayered media. - Bull. Seis. Soc. Amer., vol.43, pp.17-34.

Hunter S.C. (1970): Viscoelastic Waves, Progress in Solid Mechanics. - I. (ed: Sneddon I.N. and Hill R.) Cambridge University Press.

Jeffreys H. (1970): The Earth. - Cambridge University Press. 
Jones J.P. (1964): Wave propagation in a two layered medium. - J. Appl. Mechanics, pp.213-222.

Lamb H. (1926): On waves in elastic plate. - Proc. R. Soc. (London) A93, pp.114-128.

Love A.E.H. (1965): Some Problems of Geodynamics. - London: Cambridge University Press.

Paria G. (1960): Love waves in granular medium. - Bull. Calcutta. Math. Soc.52, No.4, pp.195-203.

Roy P.P. (1984): Wave propagation in a think two layered medium with stress couples under initial stresses. - Acta Mechanics, vol.54, pp.1-21.

Sengupta P.R. and Acharya D. (1979): The influence of gravity on the propagation of waves in a thermoelastic layer.Rev. Romm. Sci. Techmol. Mech. Appl., vol.24, pp.395-406.

Sezawa K. (1927): Dispersion of elastic waves propagated on the surface of stratified bodies and on curved surfaces. Bull. Earthq. Res. Inst. Tokyo, vol.3, pp.1-18.

Sharma J.N. and Kaur D. (2010): Rayleigh waves in rotating thermoelastic solids with voids. - Int. J. of Appl. Math. and Mech., vol.6(3), pp.43-61.

Stoneley R. (1924): Elastic waves at the surface of separation of two solids. - Proc. R. Soc. A, vol.806, pp.416-428.

Voigt W. (1887): Theoretische studien über die elasticitätsverhältnisse der krystalle. - Abh. Gesch. Wiss, vol.34, pp.351.

Received: May 20, 2012

Revised: June 26, 2013 